\section{Triagem neonatal para hemoglobinopatias: experiência de um ano na rede de saúde pública do Rio Grande do Sul, Brasil}

\author{
Neonatal screening for hemoglobinopathies: \\ a one-year experience in the public health \\ system in Rio Grande do Sul State, Brazil
}

Camila K. Sommer 1 Ana Stela Goldbeck 2 Sandrine C.Wagner 3 Simone M. Castro ${ }^{1,2}$

\footnotetext{
1 Hospital de Clínicas de Porto Alegre, Universidade Federal do Rio Grande do Sul, Porto Alegre, Brasil. 2 Faculdade de Farmácia, Universidade Federal do Rio Grande do Sul, Porto Alegre, Brasil. 3 Centro Universitário Feevale, Novo Hamburgo, Brasil.

Correspondência S. M. Castro Departamento de Análises, Faculdade de Farmácia, Universidade Federal do Rio Grande do Sul. Av. Ipiranga 2752, sala 303 Porto Alegre, RS 90610-000, Brasil. scastro@adufrgs.ufrgs.br
}

\section{Abstract}

Hemoglobinopathies are the most common inherited disorders in humans; the most frequent are hemoglobins $S$ and $C$ ( $H b S$ and $H b C$ ). Studies in Brazil show the high prevalence of $\mathrm{Hb} S$ and $\mathrm{Hb} \mathrm{C}$ heterozygotes, indicating the need for diagnosis to allow medical care and suitable genetic counseling. This study evaluates the prevalence of hemoglobin patterns in different areas of Rio Grande do Sul State, Brazil, using neonatal data from the public health system. Blood samples from 117,320 newborns, obtained by heel stick, and 2,389 blood samples from parents of newborns, obtained by venopuncture, were submitted to IEF and HPLC. Among the newborns, 1,629 (1.4\%) showed abnormal hemoglobin patterns: 1,342 FAS; 225 FAC; 45 FAD; 2 FSC; 1 FS and 26 variant hemoglobin heterozygotes. We conclude that the methods used are able to identify a broad variety of hemoglobin patterns with high specificity and sensitivity. The information is of paramount importance for transmitting knowledge in the public health field, besides facilitating planning and resource allocation.

Hemoglobinopathies; Sickle Cell Anemia; Neonatal Screening

\section{Introdução}

As hemoglobinopatias resultam de mutações nos genes que codificam as cadeias globínicas alfa $(\alpha)$ e beta $(\beta)$ da molécula de hemoglobina. Com padrão de herança autossômico recessivo, são as desordens hereditárias mais comuns nos seres humanos 1, afetando aproximadamente $7 \%$ da população mundial 2 .

Atualmente, já foram descritas mais de 1.200 mutações nos genes das cadeias globínicas 3 . No entanto, as mais freqüentes e clinicamente significantes são as variantes estruturais para hemoglobinas S e C (Hb S e Hb C) 4.

A Hb S é formada por uma mutação de ponto na posição 6 do gene da globina $\beta$, trocando o ácido glutâmico por uma valina 5 . Esta variante estrutural exerce seu efeito através da precipitação e polimerização da $\mathrm{Hb} \mathrm{S}$, acarretando mudança de forma, o que conduz à deformação da hemácia, que adquire a forma de foice, aumentando a viscosidade sangüínea com formação de cristais tactóides ${ }^{6}$. Pelo seqüestro esplênico das hemácias deformadas, observase uma anemia hemolítica crônica. As manifestações clínicas são observadas apenas nos indivíduos homozigotos para a $\mathrm{Hb} \mathrm{S}$ (Hb SS), resultando na anemia falciforme 7 . Os heterozigotos (Hb AS) são classificados como portadores de traço falciforme e são geralmente assintomáticos, apresentando sintomas apenas em casos onde há diminuição da pressão parcial 
de oxigênio 8. A importância do seu diagnóstico é para o aconselhamento genético da população afetada 7 .

A hemoglobina $\mathrm{C}(\mathrm{Hb} \mathrm{C})$ é formada pela mutação de ponto na posição 6 do gene da cadeia globínica $\beta$, trocando o ácido glutâmico por uma lisina 5,9. O quadro clínico proporcionado pela $\mathrm{Hb} \mathrm{C}$ deve-se à sua capacidade de induzir a desidratação do eritrócito e a formação intracelular de cristais 9. Apenas os indivíduos homozigotos (Hb CC) apresentam sintomatologia, caracterizada por anemia hemolítica de leve a moderada. A dupla heterozigose $\mathrm{Hb}$ / C leva a uma desordem falciforme grave, apesar de ser mais leve que a anemia falciforme, justificando a importância no diagnóstico dos indivíduos heterozigotos 7 . A tendência da $\mathrm{Hb} \mathrm{C}$ cristalizar induz à perda de potássio e de água pela célula, aumentando a concentração intracelular de hemoglobina e elevando a probabilidade da $\mathrm{Hb} S$ polimerizar 9 .

A população brasileira caracteriza-se por apresentar grande heterogeneidade genética, oriunda da contribuição que lhes deram os seus grupos raciais formadores e dos diferentes graus de miscigenação nas várias regiões do país 5 . $\mathrm{O}$ maior estudo de prevalência e distribuição de hemoglobinopatias realizado no Brasil, em 1987 10 , indicou que $3,08 \%$ dos indivíduos analisados apresentavam padrões hemoglobínicos alterados, e destes, 2,49\% eram variantes estruturais. A condição $\mathrm{Hb}$ AS foi a mais prevalente, representando $60,95 \%$ do total dos portadores, enquanto que a condição $\mathrm{Hb}$ AC foi detectada em 14,27\% dos casos. A freqüência de Hb SS na população foi de $0,04 \% 10$. Estudos realizados na Bahia, Brasil, onde se encontra uma significante heterogeneidade étnica, demonstram que a freqüência de $\mathrm{Hb}$ AS varia entre $9,8 \%$ na população geral 11 e 15,4\% nos afro-descendentes 12 . No Estado de Pernambuco, Brasil, observou-se que $0,6 \%$ dos recém-nascidos analisados eram portadores de $\mathrm{Hb} \mathrm{AC}$ e 5,3\% apresentavam $\mathrm{Hb} \mathrm{S}$; deste último porcentual, 97,1\% tinham a forma $\mathrm{Hb}$ AS e 2,9\% eram portadores de Hb SC 6 . No Estado de São Paulo, Brasil, um programa de triagem neonatal encontrou uma freqüência de $1,98 \%$ para $\mathrm{Hb} \mathrm{AS}, 0,57 \%$ para $\mathrm{Hb} \mathrm{AC}$ e $0,01 \%$ para Hb SS 13. Em Minas Gerais observou-se a incidência de variantes estruturais em 3,6\% dos indivíduos analisados e foi observado um caso de Hb SC para cada 3.450 recém-nascidos 14 . Um estudo piloto para triagem neonatal de hemoglobinopatias, realizado em Porto Alegre, Rio Grande do Sul, Brasil, indicou que 2,5\% dos indivíduos analisados tinham resultados alterados; destes, $1,2 \%$ era portador do gene para $\mathrm{Hb}$ $\mathrm{S}$, e $0,4 \%$ eram portadores do gene da Hb C 15 .
Em 2001, o Ministério da Saúde incluiu a pesquisa de hemoglobinopatias no Programa Nacional de Triagem Neonatal (PNTN), por meio da Portaria $n$. 822/01, reconhecendo a sua relevância na saúde pública do Brasil 16. O diagnóstico e o tratamento precoces aumentam a sobrevida dos afetados e melhoram a sua qualidade de vida. Além disso, o aconselhamento genético, em um contexto educativo, pode contribuir para reduzir a incidência dessas alterações 16 .

Este estudo teve por objetivo avaliar a prevalência dos padrões hemoglobínicos em diferentes regiões do Estado do Rio Grande do Sul. Este dado torna-se de extrema relevância para, em concordância com o já realizado em outros estados, determinar a prevalência de indivíduos portadores em homozigose e heterozigose de hemoglobinas variantes em nossa população, e assim, tomar as condutas clínicas e de aconselhamento familiar pertinentes.

\section{Material e métodos}

Foi realizado um estudo retrospectivo por meio da análise dos resultados dos exames de recém-nascidos atendidos pelo Sistema Único de Saúde (SUS), rastreados no Laboratório de Referência em Triagem Neonatal, Faculdade de Farmácia, Universidade Federal do Rio Grande do Sul, no período de outubro de 2003 a setembro de 2004. As amostras de sangue capilar dos 117.320 recém-nascidos obtidas em papel filtro foram analisadas através dos métodos de Cromatografia Líquida de Alta Pressão (HPLC; Bio-Rad Variant Hemoglobin Testing System Sickle Cell Short Program) e Focalização Isoelétrica (FIE; Perkin Elmer) 17. As amostras de sangue dos pais foram analisadas por FIE e HPLC (Bio-Rad $\beta$-Thal Short Program).

Analisou-se o padrão hemoglobínico de todos os recém-nascidos que se submeteram à triagem neonatal. Os pais dos recém-nascidos que apresentaram padrão hemoglobínico alterado foram convocados para confirmação do resultado. Os perfis foram classificados segundo os padrões $\mathrm{Hb} \mathrm{AA}, \mathrm{Hb} \mathrm{AS}, \mathrm{Hb} \mathrm{AC}, \mathrm{Hb} \mathrm{AD}, \mathrm{Hb}$ SS, Hb SC e Hb Var (variantes não identificadas). Salienta-se que, no período da coleta das amostras dos recém-nascidos, o padrão considerado normal é Hb FA, uma vez que a Hb Fetal encontra-se com seus valores ainda elevados.

Os dados foram agrupados segundo a divisão regional do Estado do Rio Grande do Sul, conforme estabelecido pelo Instituto Brasileiro de Geografia e Estatística (IBGE): região noroeste rio-grandense, região nordeste rio-grandense, região metropolitana de Porto Alegre, 
região sudeste rio-grandense, região centrooriental rio-grandense, região centro-ocidental rio-grandense e região sudoeste rio-grandense. Foram obtidos o número total de exames realizados e o número de exames com resultado alterado, durante o período em estudo, estratificados por mês e por região.

Os dados obtidos foram analisados utilizando-se os pacotes estatísticos Epi Info versão 6.0 (Centers for Disease Control and Prevention, Atlanta, Estados Unidos) e SPSS versão 11.0 (SPSS Inc., Chicago, Estados Unidos).

O projeto de pesquisa foi previamente aprovado pela Comissão de Pesquisa (COMPESQ), Faculdade de Farmácia, Universidade Federal do Rio Grande do Sul, e desenvolvido integralmente segundo os princípios éticos estabelecidos na Resolução n. 196/96 do Conselho Nacional de Saúde.

\section{Resultados}

No período estudado foi realizada a triagem neonatal em 117.320 recém-nascidos, sendo que destes, $1.629(1,4 \%)$ apresentaram padrão hemoglobínico alterado (Tabela 1).

A Tabela 2 apresenta a distribuição de hemoglobinas alteradas em cada região do estado.

Os pais dos recém-nascidos que apresentaram padrão hemoglobínico diferente de $\mathrm{Hb}$ FA foram convocados, sendo que 1.253 mães e 1.136 pais retornaram para a determinação de seu padrão hemoglobínico (Tabela 3). Analisando-se a possibilidade dessas famílias em gerar um indivíduo afetado (pais heterozigotos para hemoglobinas), 28 (1,7\%) apresentaram risco, sendo que em 501 famílias $(30,8 \%)$ não foi possível a determinação do mesmo, pois a mãe e/ou o pai não compareceram para confirmação do resultado (Tabela 4).

A prevalência com que as $\mathrm{Hb} \mathrm{S}$ e $\mathrm{Hb} \mathrm{C}$ (homozigose e heterozigose) ocorreram em cada região foi avaliada (Tabela 5) e os dados mostram que não houve diferença estatisticamente significativa entre as regiões.

\section{Discussão}

Os benefícios do diagnóstico e da intervenção precoce no acompanhamento das doenças falciformes têm levado à ampla difusão em todo mundo de programas para a detecção dessas condições 13. Por meio dos programas de triagem neonatal, consegue-se reduzir as taxas de morbidade e mortalidade nos primeiros cinco anos de vida 4. Além disso, o uso profilático de
Tabela 1

Prevalência de hemoglobinas alteradas

nos recém-nascidos. Rio Grande do Sul, Brasil.

\begin{tabular}{lrc}
\hline Perfil Hb & Número & Prevalência \\
\hline Hb FAS & 1.342 & $1: 87$ \\
Hb FAC & 225 & $1: 521$ \\
Hb FAD & 45 & $1: 2.600$ \\
Hb Var & 14 & $1: 8.380$ \\
Hb FSC & 2 & $1: 58.660$ \\
Hb FS & 1 & $1: 117.320$ \\
Total & 1.629 & \\
\hline
\end{tabular}

$\mathrm{Hb}$ Var = hemoglobina variante não identificada.

Tabela 2

Prevalência de hemoglobinas alteradas, por região. Rio Grande do Sul, Brasil.

\begin{tabular}{lccc}
\hline Região & N & $\begin{array}{c}\text { Hemoglobinas alteradas } \\
\text { \% }\end{array}$ \\
\hline Metropolitana & 55.450 & 868 & 1,6 \\
Noroeste & 19.981 & 153 & 0,8 \\
Sudoeste & 10.217 & 167 & 1,6 \\
Sudeste & 9.785 & 178 & 1,8 \\
Centro-oriental & 9.249 & 107 & 1,2 \\
Nordeste & 6.776 & 78 & 1,2 \\
Centro-ocidental & 5.862 & 78 & 1,3 \\
Total & 117.320 & 1.629 & 1,4 \\
\hline
\end{tabular}

Tabela 3

Padrão hemoglobínico dos pais. Rio Grande do Sul, Brasil.

\begin{tabular}{lrrrr}
\hline Hb & \multicolumn{2}{c}{ Pai } & \multicolumn{2}{c}{ Mãe } \\
& $n$ & \multicolumn{1}{c}{$\%$} & $n$ & $\%$ \\
\hline Hb AA & 590 & 51,93 & 597 & 47,60 \\
Hb AS & 452 & 39,80 & 552 & 44,10 \\
Hb AC & 75 & 6,60 & 84 & 6,70 \\
Hb AD & 13 & 1,14 & 10 & 0,80 \\
Hb Var & 3 & 0,26 & 9 & 0,72 \\
Hb SC & 3 & 0,26 & 0 & 0,00 \\
Hb SS & 0 & 0,00 & 1 & 0,08 \\
Total & 1.136 & 100,00 & 1.253 & 100,00 \\
\hline
\end{tabular}

$\mathrm{Hb}$ Var = hemoglobina variante não identificada. 
penicilina, a administração de vacina antipneumococo e outros cuidados intensivos 18, aumentam significativamente a sobrevida e a qualidade de vida dos portadores das doenças falciformes, diminuem as seqüelas e atenuam as complicações clínicas 16 .

Com a introdução da investigação de hemoglobinas variantes no PNTN, no ano de 2001, iniciou-se um trabalho de identificação do padrão hemoglobínico dos recém-nascidos, assim como de seus pais e familiares próximos. Apesar desta oportunidade, aproximadamente $25 \%$ das famílias não retornaram para fazer o teste confirmatório, dificultando o processo de triagem e identificação da totalidade que tem acesso ao teste disponibilizado pela rede de saúde pública.

A Hb S é a desordem hereditária mais comum conhecida nos seres humanos. Nossos resultados mostraram uma baixa prevalência das doenças falciformes (Hb SS e Hb SC) (1:39.000 ou $0,0025 \%$ ), quando comparadas com as outras doenças rastreadas pelo Programa Estadual de Triagem Neonatal (PETN) como hipotireoidismo congênito primário (HCP) e fenilce-

Tabela 4

Famílias em risco de gerar um filho afetado. Rio Grande do Sul, Brasil.

\begin{tabular}{lrc}
\hline Risco & N & \% \\
\hline Sim & 28 & 1,7 \\
Não & 1.100 & 67,5 \\
Desconhecido & 501 & 30,8 \\
\hline
\end{tabular}

Tabela 5

Prevalência regional de Hb S e Hb C. Rio Grande do Sul, Brasil.

\begin{tabular}{lrrrr}
\hline Região & \multicolumn{2}{c}{ Hb S } & \multicolumn{2}{c}{ Hb C } \\
& $n$ & $\%$ & $n$ & $\%$ \\
\hline Noroeste & 114 & 74,5 & 30 & 19,6 \\
Nordeste & 61 & 78,2 & 10 & 12,8 \\
Metropolitana & 721 & 83,1 & 117 & 13,5 \\
Sudeste & 151 & 84,8 & 23 & 12,9 \\
Centro-oriental & 86 & 80,4 & 15 & 14,0 \\
Centro-ocidental & 64 & 82,1 & 9 & 11,5 \\
Sudoeste & 145 & 86,8 & 21 & 12,6 \\
\hline
\end{tabular}

Hb S: $\chi^{2}=11,06 ;$ g.l. $=6 ; p=0,08652$

$\mathrm{Hb} \mathrm{C}: \chi^{2}=5,14 ; \mathrm{g} . \mathrm{l} .=6 ; \mathrm{p}=0,52619$. tonúria (PKU) que apresentaram, respectivamente, prevalências de 1:2.307 e 1:16.483. Outras regiões do Brasil, com forte imigração africana, tais como Sudeste e Nordeste, apresentaram prevalências de doença falciforme superiores às nossas na população afro-descendente $(0,1$ a $0,3 \%) 6$. As diferentes prevalências dessas hemoglobinopatias, nas diferentes regiões, refletem a diversidade de origens raciais e os variados graus de miscigenação presentes no Brasil.

Estudos brasileiros demonstraram alta prevalência de heterozigotos para hemoglobina em indivíduos afro-descendentes, variando de 6,9 a $15,4 \% 11$. Em nosso estudo, identificamos a prevalência da heterozigose em 0,73\% (1:72) do total de recém-nascidos testados, representando 99\% de todos os resultados com padrão hemoglobínico anormal. A detecção de heterozigotos é uma informação genética importante para identificar famílias que possuem risco de gerar crianças portadoras de doença falciforme, possibilitando o acesso desta família ao aconselhamento familiar.

Os genes de $\mathrm{Hb} \mathrm{S}$ e $\mathrm{Hb} \mathrm{C}$ identificados na amostra apresentaram-se distribuídos de forma homogênea entre as diferentes regiões atendidas pelo PETN, não havendo uma diferença significativa no número de ocorrências em cada região testada. Atualmente, o PETN alcança uma cobertura na rede pública de $72 \%$ do recém-nascidos vivos que fazem o teste de triagem neonatal no estado. Somando-se àqueles realizados pela iniciativa privada, a cobertura atinge $92 \%$ dos recém-nascidos vivos. Os fatores que impedem uma cobertura completa ainda precisam ser melhor estudados pelos órgãos públicos.

A detecção de um recém-nascido com padrão hemoglobínico alterado desencadeia uma cascata de testes nos demais membros da família, demonstrando um dos benefícios adicionais da triagem neonatal, possibilitando a detecção de doença e a investigação e o aconselhamento de outros membros da família. Em nosso estudo, encontramos três pais com o perfil Hb SC e uma mãe com perfil Hb SS, que puderam realizar tratamento para aliviar sintomas e melhorar sua qualidade de vida. Além disso, a presença de 14 amostras classificadas como variantes demonstra a existência de outras hemoglobinas em nosso meio, ainda não identificadas corretamente pela falta de estudos mais avançados, tais como reação da cadeia polimerase, seqüenciamento e espectrometria de massa $19,20,21$.

Além dos benefícios citados anteriormente, podemos inferir que a inclusão do rastreamen- 
to neonatal da anemia falciforme e outras hemoglobinopatias em um programa de triagem populacional, amparado pelo Ministério da Saúde, traz à luz e discute de forma ampla questões adjacentes à questão diagnóstica. Essas questões remetem a uma perspectiva êmica da etnicidade e seus desdobramentos, bem como à promoção da acessibilidade à atenção básica

\section{Resumo}

As hemoglobinopatias são as desordens hereditárias mais comuns nos seres humanos, sendo que as mais freqüentes são as hemoglobinas $\mathrm{S}$ e C ( $\mathrm{Hb} \mathrm{S}$ e Hb C). Estudos realizados no Brasil mostram a alta prevalência de heterozigotos para $\mathrm{Hb} \mathrm{S}$ e $\mathrm{Hb}$, indicando a necessidade de seu diagnóstico, permitindo o atendimento médico e o aconselhamento genético adequados. $O$ presente estudo avaliou a prevalência dos padrões hemoglobínicos em diferentes regiões do Estado do Rio Grande do Sul, Brasil, obtidos de recém-nascidos atendidos pela rede de saúde pública. Amostras de sangue coletadas sob papel filtro de 117.320 recém-nascidos e de 2.389 pais de recém-nascidos foram avaliadas por FIE e HPLC. Dentre os recém-nascidos, 1.629 (1,4\%) apresentaram padrão hemoglobínico alterado: 1.342 FAS, 225 FAC, 45 FAD, 2 FSC, 1 FS e 26 heterozigotos para variantes raras. Concluiu-se que os métodos utilizados são capazes de identificar com alta especificidade e sensibilidade uma grande variedade de padrões hemoglobínicos. As informações são importantes para tornar possível o planejamento e alocação de recursos, além de ser um mecanismo de transmissão de conhecimentos para profissionais de saúde.

Hemoglobinopatias; Anemia Falciforme; Triagem Neonatal em saúde de uma população historicamente desassistida e economicamente menos favorecida. A complementaridade entre as dimensões analisadas, portanto, justifica em um caráter de mais valia o custo efetividade deste programa, tornando-se importante em termos de planejamento administrativo, na alocação de recursos e no planejamento de políticas públicas.

\section{Colaboradores}

C. K. Sommer foi responsável pela pesquisa bibliográfica, alimentação do banco de dados, análise estatística e redação do artigo. A. S. Goldbeck participou do fornecimento e organização das informações, revisão bibliográfica e revisão da redação do artigo. S. C. Wagner e S. M. Castro realizaram testes confirmatórios para Hbs variantes, revisão dos dados e redação do artigo. S. C. Wagner também trabalhou na estruturação do banco de dados. 


\section{Referências}

1. Davies SC, Cronin E, Gill M, Greengross P, Hickman M, Normand C. Screening for sickle cell disease and thalassaemia: a systematic review with supplementary research. Health Technol Assess 2000; 4:i-v, 1-99.

2. Patrinos GP, Giardine B, Riemer C, Miller W, Chui DHK, Anagnou NP, et al. Improvements in the HbVar database of human hemoglobin variants and thalassemia mutations for population and sequence variation studies. Nucleic Acids Res 2004; 32 (Database issue):D537-41.

3. Globin Gene Server. HbVar: a database of human hemoglobin variants and thalassemias. http:// globin.cse.psu.edu/globin/hbvar/ (acessado em 13/Jan/2005).

4. Almeida AM, Henthorn JS, Davies SC. Neonatal screening for haemoglobinopathies: the results of a 10-year programme in an English Health Region. Br J Haematol 2001; 112:32-5.

5. Naoum PC. Hemoglobinopatias e talassemias. São Paulo: Editora Sarvier; 1999.

6. Bandeira FMGC, Leal MC, Souza RR, Furtado VC, Gomes YM, Marques NM. Características de recémnascidos portadores de hemoglobina "S" detectados através de triagem em sangue de cordão umbilical. J Pediatr (Rio de J) 1999; 75:167-71.

7. Clarke GM, Higgins TN. Laboratory investigation of hemoglobinopathies and thalassemias: review and update. Clin Chem 2000; 46:1284-90.

8. Iñígueza ED, López MAC, Cela de Julián ME, García PG. Detección precoz neonatal de anemia falciforme y otras hemoglobinopatías en la comunidad autónoma de Madrid. Estudio Piloto. An Pediatr 2003; 58:146-55.

9. Nagel RL, Fabry ME, Steinberg MH. The paradox of hemoglobin SC disease. Blood Rev 2003; 17:16778.

10. Naoum PC, Alvares F, Bonini-Domingos CR, Ferrari F, Moreira HW, Sampaio ZA, et al. Hemoglobinas anormais no Brasil: prevalência e distribuição geográfica. Rev Bras Patol Clín 1987; 23:68-79.

11. Azevedo ES, Alves AFP, Silva MCBO, Souza MGF, Lima AMVMD, Azevedo W. Distribution of abnormal hemoglobins and glucose-6-phosphate dehydrogenase variants in 1200 school children of Bahia, Brazil. Am J Phys Anthropol 1980; 53:509-12.
12. Adorno EV, Couto FD, Moura Neto JP, Menezes JF, Rêgo M, Reis MG, et al. Hemoglobinopathies in newborns from Salvador, Bahia, Northeast Brazil. Cad Saúde Pública 2005; 21:292-8.

13. Brandelise S, Pinheiro V, Gabetta CS, Hambleton I, Serjeant B, Serjeant G. Newborn screening for sickle cell disease in Brazil: the Campinas experience. Clin Lab Haematol 2004; 26:15-9.

14. Paixão MC, Cunha Ferraz MH, Januário JN, Viana MB, Lima JM. Reliability of isoelectrofocusing for the detection of $\mathrm{HbS}, \mathrm{HbC}$, and $\mathrm{HbD}$ in a pioneering population-based program of newborn screening in Brazil. Hemoglobin 2001; 25:297-303.

15. Daudt LE, Zechmaister D, Portal L, Camargo Neto E, Silla LMR, Giugliani R. Triagem neonatal para hemoglobinopatias: um estudo piloto em Porto Alegre, Rio Grande do Sul, Brasil. Cad Saúde Pública 2002; 18:833-41.

16. Ramalho AS, Magna LA, Paiva-e-Silva RB. A Portaria no 822/01 do Ministério da Saúde e as peculiaridades das hemoglobinopatias em saúde pública no Brasil. Cad Saúde Pública 2003; 19:1195-9.

17. Campbell M, Henthorn JS, Davies SC. Evaluation of cation-exchange HPLC compared with isoelectric focusing for neonatal hemoglobinopathy screening. Clin Chem 1999; 45:969-75.

18. Henthorn JS, Almeida AM, Davies SC. Neonatal screening for sickle cell disorders. Br J Haematol 2004; 124:259-63.

19. Riou J, Godart C, Hurtrel D, Mathis M, Bimet C, Préhu C, et al. Cation-exchange HPLC evaluated for presumptive identification of hemoglobin variants. Clin Chem 1997; 43:34-9.

20. Clark BE, Thein SL. Molecular diagnosis of haemoglobin disorders. Clin Lab Haematol 2004; 26: 159-76.

21. Caruso D, Crestani M, Mitro N, da Riva L, Mozzi R, Sarpau S, et al. High pressure liquid chromatography and electrospray ionization mass spectrometry are advantageously integrated into a two-levels approach to detection and identification of haemoglobin variants. Clin Lab Haematol 2005; 27:111-9.

Recebido em 12/Mai/2005

Versão final reapresentada em 06/Set/2005 Aprovado em 08/Nov/2005 